\title{
Update on fruit antioxidant capacity: a key tool for Mediterranean diet
}

\author{
Maurizio Battino ${ }^{1, *}$ and Bruno Mezzetti ${ }^{2}$ \\ ${ }^{1}$ Faculty of Medicine, Institute of Biochemistry, Università Politecnica delle Marche-Ancona, Via Ranieri, 65, 60127 \\ Ancona, Italy: ${ }^{2}$ SAPROV, Department of Environmental and Crop Science, Faculty of Agriculture, Università \\ Politecnica delle Marche-Ancona, Ancona, Italy
}

\section{Submitted 4 April 2006: Accepted 11 0ctober 2006}

\begin{abstract}
Objective: To review and update the most relevant research dealing with the antioxidant properties of fruits which can be useful in Mediterranean and other healthy diets.

Design: Personal perspectives and late data.

Setting: International

Results: The evaluation of total antioxidant capacity of fruits is of pivotal importance in assessing the nutritional attributes of these products. By means of specific breeding programmes, it is possible to select antioxidant-enriched fruit varieties. These features are susceptible to be improved through generations in order to release fruits with enhanced nutritional features.

Conclusions: The availability of high quality and nutritionally enriched fruit at competitive costs may be a useful tool in the planning of healthy diets.
\end{abstract}

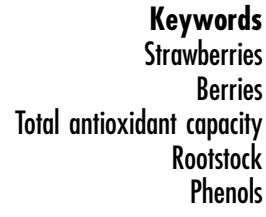

Keywords

Berries

Rootstock

Phenols
The consumer is continuously looking for a diet containing a large array of compounds that may function as antioxidants, including vitamins $\mathrm{E}$ and $\mathrm{C}$, carotenoids, flavonoids and other phenolic compounds, and that may be helpful in the daily challenge against free radical and reactive oxygen species which are produced (often) physiologically by our tissues ${ }^{1}$.

Consumers, in the last few years, have paid increasing attention to the health and nutritional aspects (vitamins contents, mineral elements, antioxidants, etc.) of horticultural products. A diet rich in fruits and vegetables, like the Mediterranean diet, offers protection against some common diseases, such as cardiovascular events, cancer and other age-related degenerative diseases ${ }^{2}$. Fruits have long been regarded as having considerable health benefits, particularly due to their antioxidant content, which is able to protect the human body against the cellular oxidation reactions ${ }^{3,4}$.

In recent years, epidemiological studies have demonstrated an inverse association between the intake of antioxidants from fruits and vegetables and the morbidity and mortality from stroke ${ }^{5}$, cardiovascular diseases ${ }^{6}$ and cancer $^{7-9}$. The socio-economic and public health impact of all these pathologies is impressive and recently the consumer interest in 'healthy eating' has increased awareness about the importance of limiting and/or selecting the consumption of foods.

The greater consumption of fruit and vegetables can be considered as one way to increase the intake of antioxidants. A second choice can be the use of functional foods containing antioxidants and/or a limited consumption of specific and peculiar fruits, such as berries, which are extremely rich sources of compounds with antioxidant activity $^{10}$. Therefore, the increase of antioxidant levels through breeding and/or biotech is an important option to support a higher antioxidant intake even when the consumption of fruit is low.

Therefore, basic investigation and breeding work has been deeply involved in improving the quality of fruits and in selecting new varieties characterised, on the one hand, by commercially high-quality products (according to the typical parameters of firmness, colour, soluble solid content and titratable acidity) and, on the other, by tasteful and highly nutritionally enriched fruits.

\section{The current targets of agronomic research}

In the last decades, agronomic research has set priorities to obtain high yield, better resistance to disease and to transport, as well as a longer shelf life of fruit. Thus, the breeding programme of fruit took into consideration the important objectives such as improving yield and fruit size, resistance to diseases and pests, adaptation to particular growing systems as well as harvesting speeds. In general, the quality of fruit is considered an extremely complex matter because it is difficult to describe objectively. Sensory aspects like shape and colour are almost exclusively limited to the appearance of the products, 
but nowadays firmness, total acid and sugar content and nutritional values are also considered o better characterise the fruit quality. Research is now focused on a concept of fruit quality, extended also to the improvement of the nutritional value.

Nutritional value can be identified by the content of specific (antioxidant) compounds, but the overall nutritional value could be better specified by analysing the total antioxidant potential which, in turn, could depend on the specific plant genotype (type of fruit, species and variety within species ${ }^{11}$ ) and interactions of cultivation conditions (environmental and cultivation techniques). Other factors that may affect antioxidant properties include ripening season, preharvest environmental conditions, shelf life and processing.

In order to support new and complementary data to better characterise agronomic production and information to the consumer, a number of assays have been introduced in the past decade for determining both the contents of individual antioxidant molecules and the total antioxidant capacity (TAC) of fruit extracts. This latter parameter is extremely useful since TAC does not always depend directly upon antioxidant concentrations but considers the cumulative action and the synergistic interaction of all the antioxidants present in fruits giving the cumulative capacity of fruits to scavenge free radicals ${ }^{12}$. Whilst most of the TAC assays are hardly suitable for complex biological mixtures (like plasma or serum), and specific devices for avoiding masking features are required ${ }^{13}$, TAC evaluation of fruit extracts is usually easy, cheap, fast and reliable even using basic instrumentation available in all research laboratories worldwide ${ }^{14-17}$ and these properties account for the recent successes that such an approach has actually obtained both in basic research and in applied investigations.

\section{What are 'bioactive components'?}

The term 'bioactive components' is usually associated with 'functional foods' since the latter should contain the former in order to be efficacious for the improvement of consumers' health.

A general theory is emerging that bioactive components' in plants induce metabolic effects including functioning as antioxidants and switching on genes that eliminate carcinogens. Bioactive components are generally defined as compounds in foods that deliver a health benefit beyond basic nutrition (International Life Science Institute, 1999). Classical genetic, as well as transgenic, approaches are being used to increase the content of specific bioactive compounds of plants, but the ability to manipulate plant metabolism is often far ahead of our understanding of whether or how such bioactive components work. There is an increasing awareness that multiple genetic and environmental factors affect production and accumulation of bioactive compounds, but these factors are seldom taken into account when a functional food-fruit is marketed.

Antioxidants could actually be considered among the most important 'bioactive compounds' of a food. The increase in the level of antioxidants in plant foods through breeding is an important option to support a higher intake even with low fruit and vegetable consumption. This approach can succeed if the variability and heritability of the antioxidant trait is indicative that progress through breeding is feasible. Accordingly, the increase of consumption of healthier fruit is seen as an appropriate strategy for improving human health. The increase of fruit consumption is also strictly related to several factors, including fruit price, but mostly to consumer quality acceptance. As a consequence, the increase of consumer health is also strictly related to new released varieties with improved quality and antioxidant attributes.

Consumer quality acceptance is generally related to specific perceived quality attributes such as fruit shape, colour, but also sweetness, acidity, combined with aroma and flavour. Nowadays, these characteristics are the major topics of several breeding programmes and/or biotech now active on berries, and even if these quality attributes are controlled by a complex genetic background and frequently associated with negative agronomic characters, such as lesser fruit firmness, fruit size or productivity, some considerable progress has been achieved, particularly for the strawberry.

\section{Strawberries as a nutritional tool for delivering antioxidants}

The highest antioxidant potential of strawberries has been often proved $^{18}$, but only recently it has been demonstrated that the high TAC of strawberries is strongly influenced by species and cultivar ${ }^{11}$ : the wild species $F$. vesca has TAC values 2.5-fold higher than the average of the most common cultivated varieties (Fragaria $x$ ananassa). Moreover, the variability detected among the cultivated new advanced selections highlighted an existing unexploited variability in the cultivated germ plasm. On this basis, well-focused breeding programmes have been applied in order to create new varieties specifically selected for improved antioxidant potential. With the aim to improve fruit antioxidant pattern in strawberries, we recently studied six families derived from cross combinations including wild parental plants also ( $F$. virginiana glauca) (Table 1). It emerged that, probably because the most recent breeding programmes consider nutritional characters with a low priority, the genotypes rarely associate production efficiency and sensorial quality with the nutritional values of fruit (Fig. 1). It often occurs that varieties characterised by high commercial parameters (expressed, for example, as sugar/acidity ratio) are not so efficient as far as antioxidant capacity is concerned. 
Table 1 Cross combination of each of the six families employed

\begin{tabular}{|c|c|c|c|c|c|}
\hline \multirow[b]{2}{*}{ Family } & \multicolumn{2}{|r|}{ Maternal (M) } & \multirow[b]{2}{*}{$X$} & \multicolumn{2}{|c|}{ Paternal (P) } \\
\hline & Genotype & Species & & Genotype & Species \\
\hline 1 & Paros & F. $x$ ananassa & $\mathrm{X}$ & Queen Elisa & F. $x$ ananassa \\
\hline 2 & Onda & F. $x$ ananassa & $\mathrm{X}$ & AN93.371.53 & F. $x$ ananassa \\
\hline 3 & Queen Elisa & F. $x$ ananassa & $\mathrm{X}$ & Sveva & F. $x$ ananassa \\
\hline 4 & AN 94.414.52 & F. $x$ ananassa $x F$. virginiana glauca & $\mathrm{X}$ & 91.143 .5 & F. $x$ ananassa \\
\hline 5 & Sveva & F. $x$ ananassa & $\mathrm{X}$ & Patty & F. $x$ ananassa \\
\hline 6 & AN 94.414 .52 & $F . x$ ananassa $x F$. virginiana glauca & $\mathrm{X}$ & Onda & F. $x$ ananassa \\
\hline
\end{tabular}

It has also been demonstrated that the TAC levels of these strawberries directly depend on their total phenol contents (Fig. 2). The study of quality and nutritional attributes in the offspring from each of the six families confirmed the variability of these traits and the importance of the parent combinations in their possible improvement (Fig. 3). The results from local released varieties showed, for the first time, the importance of $F$. virginiana glauca genetic base in improving the nutritional quality of commercial strawberries, as already observed also for other characteristics, such as male fertility, fruit size and disease resistance.

The genetic base of the different parents used in different strawberry cross combinations has determined a concomitant improvement of both fruit commercial characteristics and TAC of the new populations (Fig. 3); this last feature is probably related to the increase of the total phenol contents of these fruits.

These results ${ }^{19}$ can be considered of particular interest to better define the varieties and breeding evaluation strategies. Nowadays, these aspects are considered highly useful for the commercial valorisation of new varieties, but mostly to select new genotypes with high fruit nutritional quality combined with yield efficiency, which now corresponds with plant adaptability, production efficiency and fruit size.

Wild species such as $F$. virginiana glauca and $F$. vesca are good sources of bioactive compounds ${ }^{11}$, but in other berry species the introduction of the wild germ plasm did not improve the nutritional quality of fruit ${ }^{20,21}$. Our results demonstrate that the use of genetic material from wild germ plasm such as $F$. virginiana glauca can significantly improve the nutritional quality of the commercial strawberry. In fact, we selected new positive transgressive segregants, identified as those offspring whose 2-year mean exceeded the higher parental mean, having improved both quality attributes and nutritional attributes. This new material can really open new perspectives in breeding strawberries for the achievement of new commercial varieties with improved nutritional quality.

\section{The rootstock effects on fruit quality and nutrition}

The effect of the rootstock, which is an important agronomic tool for controlling fruit production and quality and is capable of changing a fruit's nutritional components, was also studied to explore the possibility of increasing the low antioxidant capacity of both apricots

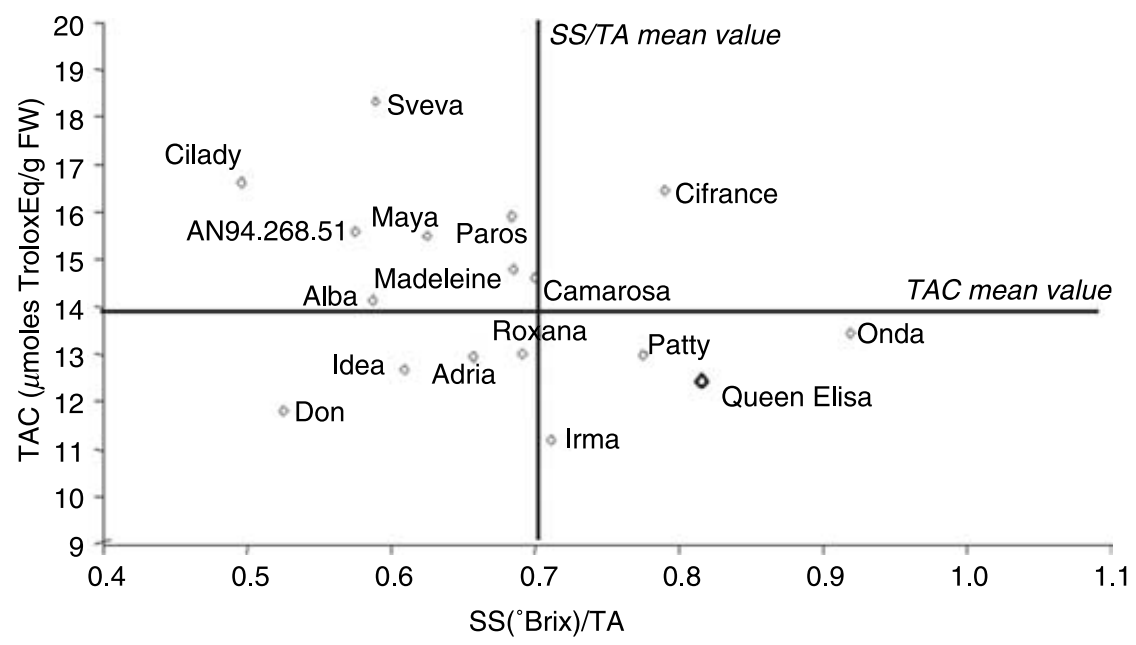

Fig. 1 Nutritional vs. commercial attributes in strawberry cultivars and selection. Nutritional characters are indicated as total antioxidant capacity (expressed in $\mu$ moles TroloxEq/g fresh weight) and commercial features by means of the SS ( ${ }^{\circ} B$ rix)/TA ratio, i.e. the ratio between the soluble solid content (expressed in ${ }^{\circ}$ Brix) and the total acidity (expressed in $\mathrm{mEq}$ of $\mathrm{NaOH}$ per $100 \mathrm{~g}$ of fruit) 


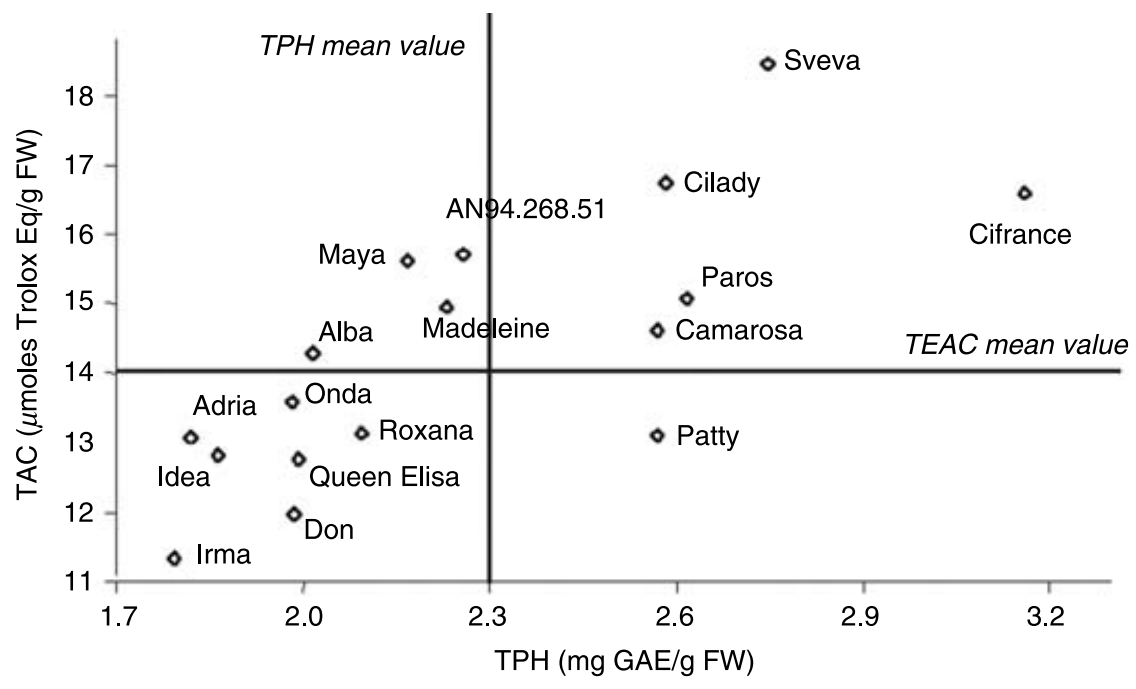

Fig. 2 Correlation between total antioxidant capacity (expressed in $\mu$ moles TroloxEq/g fresh weight) and total phenols (expressed as mg gallic acid equivalent/g fresh weight) of strawberry cultivars and selection. The Pearson's correlation coefficient resulted to be 0.57 with a $P<0.001$

and peaches ${ }^{11,14}$. Human consumption of strawberries is often limited and usually linked to a very short period of product availability, whereas apricots and peaches represent the main fruit intake during late spring and summer, representing at the same time, a major part of the standard dietary intake during a longer period of the year. Due to their features and seasonal consumption, these fruits have specific important nutritional roles such as a reservoir of minerals and water; however, they may also provide valuable levels of antioxidants. The effects induced by the rootstocks on controlling plant development and production are well known, but no data are available on the effects induced on fruit quality. The evidence obtained may suggest a clear effect of the rootstock type in determining, at constant growing conditions, nutritional variations of the same varieties of peaches and apricots. For both species, the fruit with the highest TAC was harvested from two of the currently most commonly grown rootstocks: Myrabolan 29C for apricots and GF677 for peaches. Usually, these two rootstocks graft, with a high affinity to different varieties of the two species, have a wide adaptability to different conditions and sustain the highest plant vigour (larger root and canopy development).

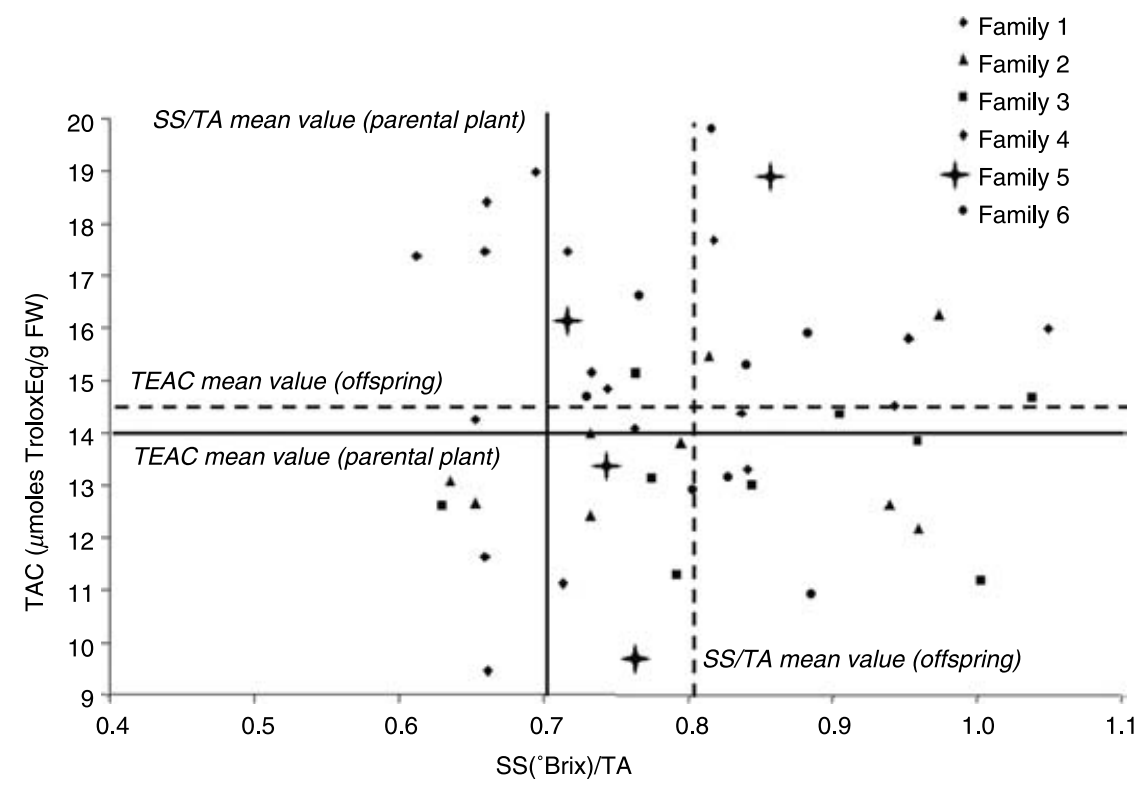

Fig. 3 Nutritional vs. commercial attributes in strawberry offspring (breeding progress). Nutritional characters are indicated as total antioxidant capacity (expressed in $\mu$ moles TroloxEq/g fresh weight) and commercial features by means of the SS ( $\left.{ }^{\circ} \mathrm{Brix}\right) / \mathrm{TA}$ ratio, i.e. the ratio between the soluble solid content (expressed in ${ }^{\circ} \mathrm{Brix}$ ) and the total acidity (expressed in mEq of $\mathrm{NaOH}$ per $100 \mathrm{~g}$ of fruit) 


\section{Conclusions}

Breeding and also biotechnological approaches are being used to increase the content of specific bioactive components of plants, but the manipulation of plant metabolism is still not that easy to address. There is an increasing awareness that multiple genetic and environmental factors affect production and accumulation of bioactive compounds, but these factors are rarely taken into account when fruit is marketed. It is possible to produce new varieties with improved nutritional values combined with high plant production efficiency and fruit quality.

Total antioxidant capacity and total phenol content values in the fruits indicate that it is possible both to select varieties with specific nutritional (antioxidant) features and that such features could be improved through generations in order to release fruits with valuable characteristics for consumers and which may be useful in assessing healthy (antioxidant-enriched) diets.

Moreover, the availability of different kinds of high quality and tasty fruit, during different seasonal periods along the year, possibly at competitive costs, may represent a useful tool for patients as well as the healthy population for planning diets containing these products; the specific enrichment of these fruit with 'bioactive substance' may also allow, in some specific cases, to reduce suggesting servings of fruits since the total recommended amount of these substances could be taken even with lower levels of fruit consumption.

\section{Acknowledgements}

Università Politecnica delle Marche-Ancona-has partially supported this work. The valuable help of Ms M Glebocki in editing the manuscript is gratefully acknowledged.

\section{References}

1 Ames BM, Shigens MK, Hagen TM. Oxidants, antioxidants and the degenerative diseases of aging. Proceedings of the National Academy of Sciences of USA 1993; 90: 7915-22.

2 Willett WC. The Mediterranean diet: science and practice. Public Health Nutrition 2006; 9(1A): 105-10.

3 Cook NC, Samman S. Flavonoids - Chemistry, metabolism, cardioprotective effects, and dietary sources. Journal of Nutritional Biochemistry 1996; 7: 66-76.

4 Cohen JH, Kristal AR, Stanford JL. Fruit and vegetable intakes and prostate cancer risk. Journal of the National Cancer Institute 2000; 92: 61-8.

5 He FJ, Nowson CA, MacGregor GA. Fruit and vegetable consumption and stroke: meta-analysis of cohort studies. Lancet 2006; 367: 320-6.

6 Hung HC, Joshipura KJ, Jiang R, Hu FB, Hunter D, SmithWarner SA, Colditz GA, Rosner B, Spiegelman D, Willett WC.
Fruit and vegetable intake and risk of major chronic disease. Journal of the National Cancer Institute 2004; 96: 1577-84.

7 Khaw KT, Bingham S, Welch A, Luben R, Wareham N, Oakes $\mathrm{S}$, Day N. Relation between plasma ascorbic acid and mortality in men and women in EPIC-Norfolk prospective study: a prospective population study. European Prospective Investigation into Cancer and Nutrition. Lancet 2001; 357: 657-63.

8 Smith-Warner SA, Spiegelman D, Yaun SS, Albanes D, Beeson WL, van den Brandt PA, Feskanich D, Folsom AR, Fraser GE, Freudenheim JL, Giovannucci E, Goldbohm RA, Graham S, Kushi LH, Miller AB, Pietinen P, Rohan TE, Speizer FE, Willett WC, Hunter DJ. Fruits, vegetables and lung cancer: a pooled analysis of cohort studies. International Journal of Cancer 2003; 107: 1001-11.

9 Etminan M, Takkouche B, Caamano-Isorna F. The role of tomato products and lycopene in the prevention of prostate cancer: a meta-analysis of observational studies. Cancer Epidemiology Biomarkers and Prevention 2004; 13: 340-5.

10 Agius F, Gonzalez-Lamothe R, Caballero JL, Monuz-Blanco J, Botella MA, Valpuesta V. Engineering increased vitamin C levels in plants by overexpression of a D-galacturonic acid reductase. Nature Biotechnology 2003; 21: 177-81.

11 Scalzo J, Politi A, Pellegrini N, Mezzetti B, Battino M. Plant genotype affects total antioxidant capacity and phenolic contents in fruit. Nutrition 2005; 21: 207-13.

12 Scalzo J, Mezzetti B, Battino M. Total antioxidant capacity evaluation: critical steps for assaying berry antioxidant features. BioFactors 2005; 23: 221-7.

13 Bompadre S, Leone L, Politi A, Battino M. Improved FIAABTS method for antioxidant capacity determination in different biological samples. Free Radical Research 2004; 38 : 831-8.

14 Giorgi M, Capocasa F, Scalzo J, Murri G, Battino M, Mezzetti B. The rootstock effects on plant adaptability, production, fruit quality and nutrition in the peach (cv. Suncreast). Scientia Horticulturae 2005; 107: 36-42.

15 Halvorsen BL, Holte K, Myhestad MCW, Barikmo I, Hvattum E, Remberg SF, Wold A-B, Haffner K, Baugerod H, Andersen LF, Moskaug JO, Jacobs DR, Blomhoff R. A systematic screening of total antioxidants in dietary plants. Journal of Nutrition 2002; 132: 461-71.

16 Pellegrini N, Re R, Yang M, Rice-Evans CA. Screening of dietary carotenoids and carotenoid-rich fruit extracts for antioxidant activities applying the $2,2^{\prime}$-azobis(3-ethylenebenzothiazoline-6-sulfonic) acid radical cation decolorization assay. Methods in Enzymology 1999; 299: 379-89.

17 Pellegrini N, Serafini M, Colombi B, Del Rio D, Salvatore S, Bianchi M, Brighenti F. Total antioxidant capacity of plant foods, beverages, and oils consumed in Italy assessed by three different in vitro assays. Journal of Nutrition 2003; 133: 2812-9.

18 Wang H, Cao G, Prior RL. Total antioxidant capacity of fruits. Journal of Agriculture and Food Chemistry 1996; 44: 701-5.

19 Scalzo J, Battino M, Costantini E, Mezzetti B. Breeding and biotechnology for improving berry nutritional quality. BioFactors 2005; 23: 213-20.

20 Connor AM, Stephens MJ, Hall HK, Alspach PA. Variation and heritabilities of antioxidant activity and total phenolic content estimated from a red raspberry factorial experiment. Journal of the American Society for Horticultural Science 2005; 130: 3 .

21 Deighton N, Brennan R, Finn C, Davies HV. Antioxidant properties of domesticated and wild Rubus species. Journal of Agriculture and Food Chemistry 2003; 80: 1307-13. 